\title{
Density Functional Theory Study of Ten-Atom Germanium Clusters: Effect of Electron Count on Cluster Geometry
}

\author{
R. B. King, ${ }^{1}$ I. Silaghi-Dumitrescu, ${ }^{2}$ and M. M. Uţă ${ }^{2}$ \\ ${ }^{1}$ Department of Chemistry, University of Georgia, Athens, Georgia, 30602 \\ ${ }^{2}$ Faculty of Chemistry and Chemical Engineering, Babeș-Bolyai University, Cluj-Napoca, Roumania
}

\section{Supporting Information}

Figure S1. $\mathrm{Ge}_{10}{ }^{2}$ Initial Structures

Table S1. Optimized $\mathrm{Ge}_{10}{ }^{z}$ Structures with their Energies and Geometries

Table S2. HOMO-LUMO energy gaps for the $\mathrm{Ge}_{10}{ }^{z}$ optimized structures (eV)

\footnotetext{
${ }^{1}$ University of Georgia

2 Babeş-Bolyai University
} 
Figure S1. $\mathrm{Ge}_{10}{ }^{2}$ Initial Structures
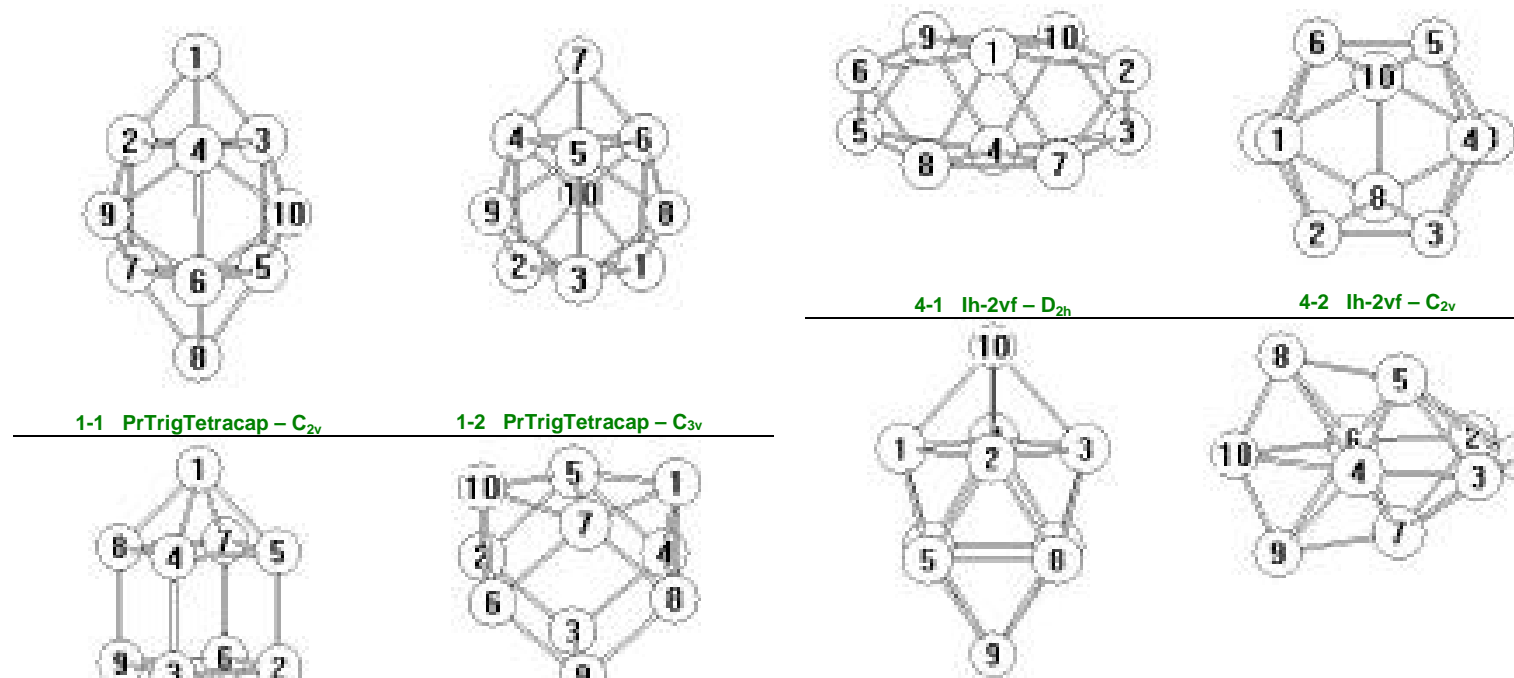

4-2 Ih-2vf $-\mathrm{C}_{2 \mathrm{v}}$

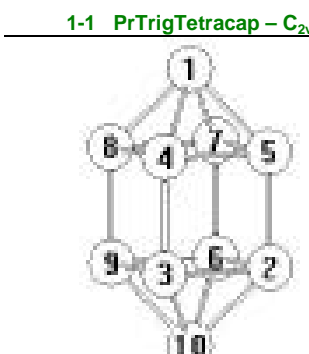

1-2 PrTrigTetracap $-\mathrm{C}_{3 \mathrm{v}}$

2-1 CubDicap - D 4 h

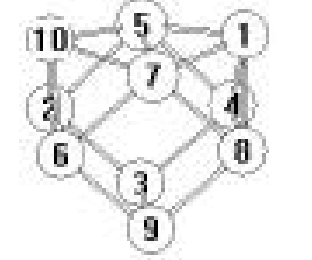

5-1 AntiPrTetraDicap - $\mathrm{D}_{4 \mathrm{~d}}$
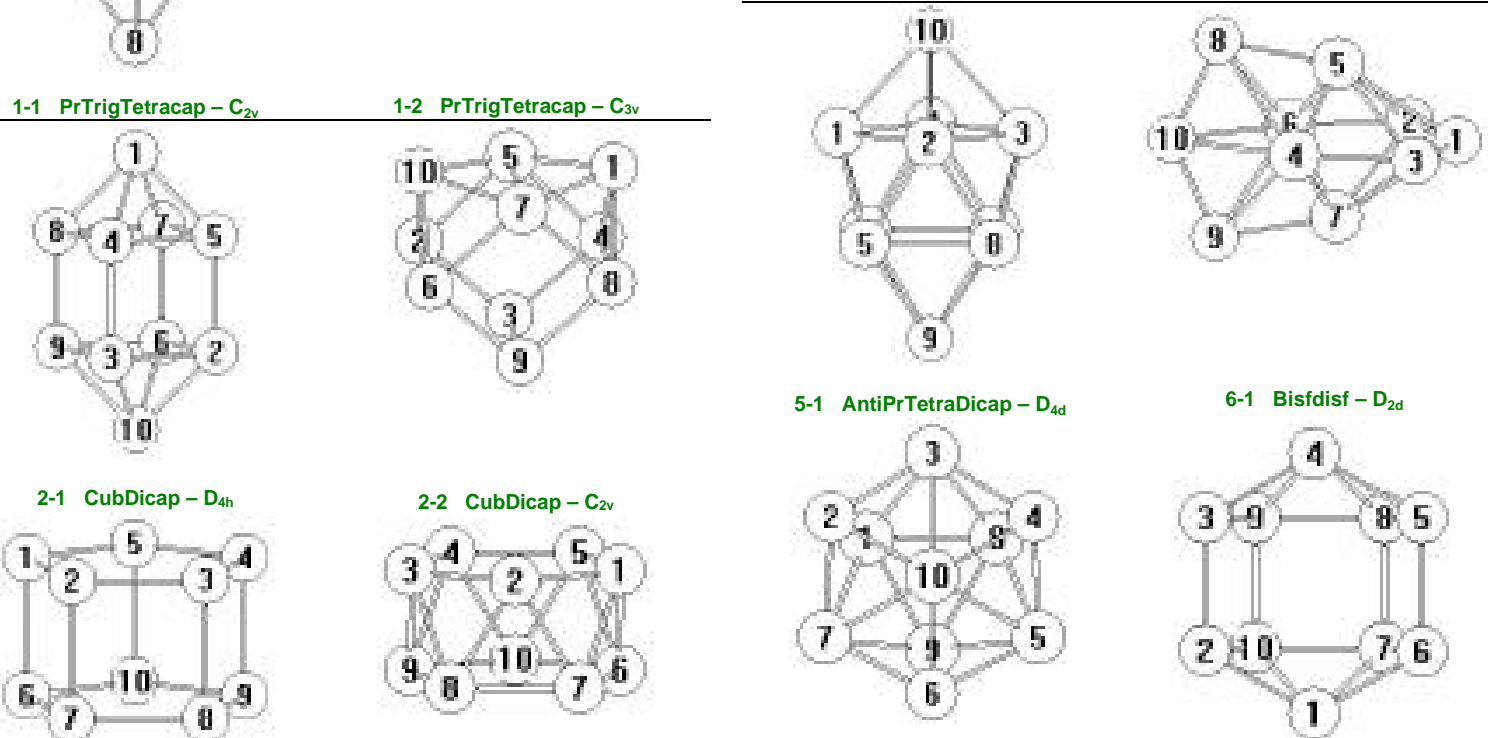

3-1 PrPentag $-D_{5 h}$

3-2 AntiPrPentag $-D_{5 d}$

7-1 Centaur $-C$

7-2 Centaur $-C_{2 v}$ 
Table S1. Optimized $\mathrm{Ge}_{10}{ }^{z}$ Structures with their Energies and Geometries

A. $\mathrm{Ge}_{10}-1-1 \_$PrTrigTetracap_ $\mathrm{C}_{2 \mathrm{v}}$

\begin{tabular}{|c|c|c|c|c|c|c|c|}
\hline $\begin{array}{c}\text { Charge } \\
6-\end{array}$ & $\begin{array}{l}1 \mathrm{Ge} \\
2 \mathrm{Ge} \\
3 \mathrm{Ge} \\
4 \mathrm{Ge} \\
5 \mathrm{Ge} \\
6 \mathrm{Ge} \\
7 \mathrm{Ge} \\
8 \mathrm{Ge} \\
9 \mathrm{Ge} \\
10 \mathrm{Ge} \\
\\
\\
6 \mathrm{Ge} \\
7 \mathrm{Ge} \\
8 \mathrm{Ge} \\
9 \mathrm{Ge} \\
10 \mathrm{Ge}\end{array}$ & $\begin{array}{c}1 \\
0.000000 \\
2.662358 \\
2.662358 \\
2.577682 \\
5.007566 \\
5.369358 \\
5.007566 \\
6.634665 \\
4.040677 \\
4.040677 \\
\\
6 \\
0.000000 \\
3.384156 \\
2.577682 \\
2.714447 \\
2.714447\end{array}$ & $\begin{array}{l}2 \\
0.000000 \\
2.920625 \\
3.384156 \\
3.985019 \\
4.529711 \\
2.711149 \\
5.007566 \\
2.663968 \\
4.321236 \\
\\
7 \\
\\
0.000000 \\
2.662358 \\
2.663968 \\
4.321236\end{array}$ & $\begin{array}{l}3 \\
\\
0.000000 \\
3.384156 \\
2.711149 \\
4.529711 \\
3.985019 \\
5.007566 \\
4.321236 \\
2.663968 \\
\\
8 \\
\\
\\
0.000000 \\
4.040677 \\
4.040677\end{array}$ & $\begin{array}{l}4 \\
\\
0.000000 \\
4.529711 \\
3.343886 \\
4.529711 \\
5.369358 \\
2.714447 \\
2.714447 \\
9 \\
\\
\\
\\
0.000000 \\
3.963657\end{array}$ & $\begin{array}{l}0.000000 \\
3.384156 \\
2.920625 \\
2.662358 \\
4.321236 \\
2.663968 \\
10\end{array}$ & $\begin{array}{l}\text { 1-1 PrTrigTetracap }-\mathrm{C}_{2 \mathrm{v}}[26-4] \\
\text { - } 20748.7290928(+28.85 \mathrm{kcal} / \mathrm{mole})\end{array}$ \\
\hline
\end{tabular}




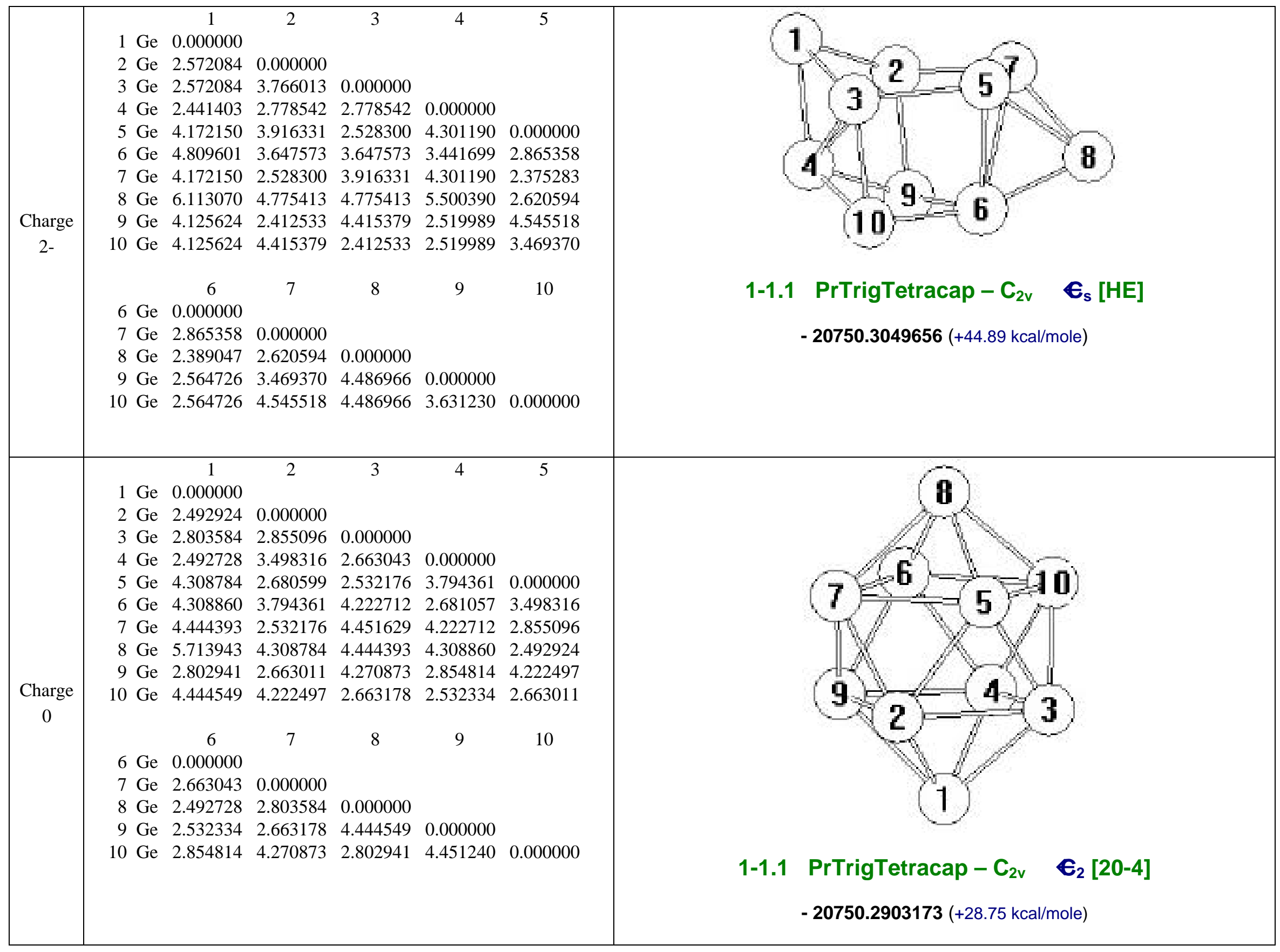




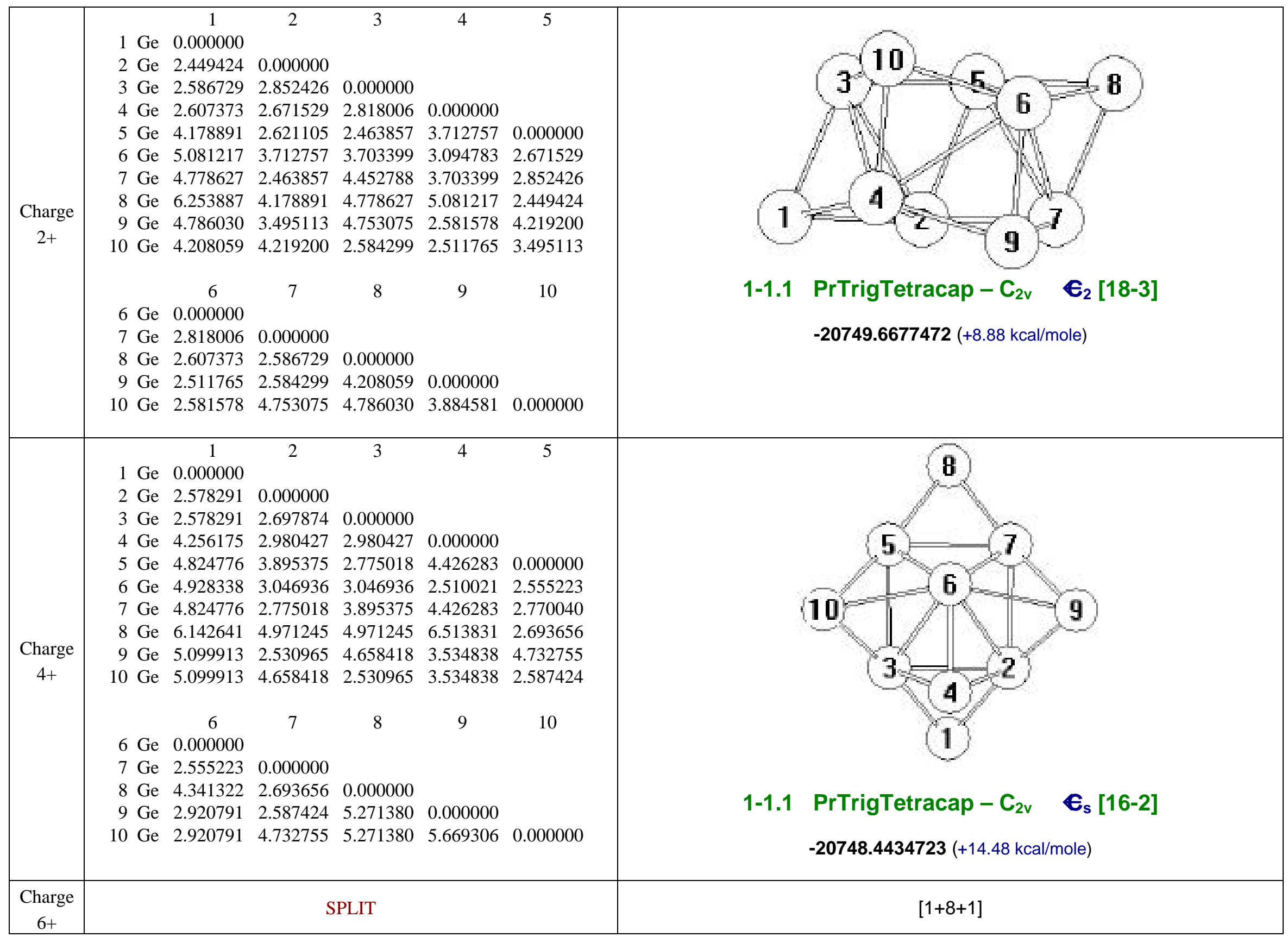


B. $\mathrm{Ge}_{10}-1-2 \_$PrTrigTetracap- $\mathrm{C}_{3 \mathrm{v}}$

\begin{tabular}{|c|c|c|c|c|c|c|c|}
\hline $\begin{array}{c}\text { Charge } \\
6-\end{array}$ & $\begin{array}{ll}1 \mathrm{Ge} \\
2 \mathrm{Ge} \\
3 \mathrm{Ge} \\
4 \mathrm{Ge} \\
5 \mathrm{Ge} \\
6 \mathrm{Ge} \\
7 \mathrm{Ge} \\
8 \mathrm{Ge} \\
9 \mathrm{Ge} \\
10 \mathrm{Ge} \\
& \\
6 \mathrm{Ge} \\
7 \mathrm{Ge} \\
8 \mathrm{Ge} \\
9 \mathrm{Ge} \\
10 \mathrm{Ge}\end{array}$ & $\begin{array}{c}1 \\
0.000000 \\
2.767967 \\
2.767967 \\
4.549240 \\
4.549240 \\
3.652406 \\
6.244018 \\
2.631267 \\
4.218880 \\
2.631267 \\
\\
6 \\
0.000000 \\
2.835187 \\
2.806475 \\
4.279854 \\
2.806475\end{array}$ & $\begin{array}{c}2 \\
0.000000 \\
2.767967 \\
3.652406 \\
4.549240 \\
4.549240 \\
6.244018 \\
4.218880 \\
2.631267 \\
2.631267 \\
7 \\
\\
0.000000 \\
4.904273 \\
4.904273 \\
4.904273\end{array}$ & $\begin{array}{c}3 \\
\\
0.000000 \\
4.549240 \\
3.652406 \\
4.549240 \\
6.244018 \\
2.631267 \\
2.631267 \\
4.218880 \\
\\
8 \\
\\
0.000000 \\
3.929016 \\
3.929016\end{array}$ & $\begin{array}{c}4 \\
\\
0.000000 \\
2.657371 \\
2.657371 \\
2.835187 \\
4.279854 \\
2.806475 \\
2.806475 \\
9\end{array}$ & $\begin{array}{c}5 \\
\\
\\
0.000000 \\
2.657371 \\
2.835187 \\
2.806475 \\
2.806475 \\
4.279854 \\
10 \\
\end{array}$ & $\begin{array}{c}\text { 1-2 PrTrigTetracap }-\mathrm{C}_{3 \mathrm{v}}[26-2] \\
-20748.7656742(+5.89 \mathrm{kcal} / \mathrm{mole})\end{array}$ \\
\hline
\end{tabular}




\begin{tabular}{|c|c|c|c|c|c|c|c|}
\hline $\begin{array}{c}\text { Charge } \\
2-\end{array}$ & $\begin{array}{l}1 \mathrm{Ge} \\
2 \mathrm{Ge} \\
3 \mathrm{Ge} \\
4 \mathrm{Ge} \\
5 \mathrm{Ge} \\
6 \mathrm{Ge} \\
7 \mathrm{Ge} \\
8 \mathrm{Ge} \\
9 \mathrm{Ge} \\
10 \mathrm{Ge} \\
\\
\\
6 \mathrm{Ge} \\
7 \mathrm{Ge} \\
8 \mathrm{Ge} \\
9 \mathrm{Ge} \\
10 \mathrm{Ge}\end{array}$ & $\begin{array}{c}1 \\
0.000000 \\
2.590198 \\
2.819760 \\
4.210715 \\
4.207954 \\
2.541375 \\
4.189871 \\
2.542620 \\
3.989551 \\
2.822604 \\
\\
6 \\
0.000000 \\
2.587741 \\
2.820403 \\
4.209902 \\
2.540637\end{array}$ & $\begin{array}{c}2 \\
0.000000 \\
2.592671 \\
4.186317 \\
4.191875 \\
4.186621 \\
5.335684 \\
4.191728 \\
2.589362 \\
2.586643 \\
\\
7 \\
\\
0.000000 \\
2.591510 \\
4.189082 \\
4.185615\end{array}$ & $\begin{array}{c}3 \\
\\
0.000000 \\
4.209011 \\
2.542777 \\
4.208603 \\
4.193488 \\
2.542913 \\
2.818069 \\
3.989454 \\
\\
8 \\
\\
\\
0.000000 \\
4.206593 \\
4.208634\end{array}$ & $\begin{array}{c}4 \\
\\
0.000000 \\
2.821592 \\
2.824387 \\
2.588776 \\
3.989628 \\
2.541404 \\
2.540777 \\
9 \\
\\
\\
\\
\\
\\
2.000000 \\
2.823587\end{array}$ & $\begin{array}{c}5 \\
\\
\\
0.000000 \\
3.988803 \\
2.591303 \\
2.816828 \\
2.541973 \\
4.209351 \\
10\end{array}$ & PrTrigTetracap $-\mathrm{C}_{3 \mathrm{v}} \quad \boldsymbol{\epsilon}_{1}[22-1]$ \\
\hline
\end{tabular}




\begin{tabular}{|c|c|c|c|c|c|c|c|}
\hline $\begin{array}{c}\text { Charge } \\
2+\end{array}$ & $\begin{aligned} 1 \mathrm{G} \\
2 \mathrm{G} \\
3 \mathrm{G} \\
4 \mathrm{G} \\
5 \mathrm{G} \\
6 \mathrm{G} \\
7 \mathrm{G} \\
8 \mathrm{G} \\
9 \mathrm{G} \\
10 \mathrm{G} \\
\\
\\
6 \mathrm{G} \\
7 \mathrm{G} \\
8 \mathrm{G} \\
9 \mathrm{G} \\
10 \mathrm{G}\end{aligned}$ & $\begin{array}{c}1 \\
0.000000 \\
3.227794 \\
3.227794 \\
3.914509 \\
3.914509 \\
2.514065 \\
4.853802 \\
2.595640 \\
4.630256 \\
2.595640 \\
\\
6 \\
0.000000 \\
2.552569 \\
2.680956 \\
4.459999 \\
2.680956\end{array}$ & $\begin{array}{c}2 \\
0.000000 \\
3.227794 \\
2.514065 \\
3.914509 \\
3.914509 \\
4.853802 \\
4.630256 \\
2.595640 \\
2.595640 \\
\\
7 \\
\\
0.000000 \\
4.269787 \\
4.269787 \\
4.269787\end{array}$ & $\begin{array}{c}3 \\
\\
0.000000 \\
3.914509 \\
2.514065 \\
3.914509 \\
4.853802 \\
2.595640 \\
2.595640 \\
4.630256 \\
\\
8 \\
\\
\\
0.000000 \\
4.554789 \\
4.554789\end{array}$ & $\begin{array}{c}4 \\
\\
\\
0.000000 \\
2.789168 \\
2.789168 \\
2.552569 \\
4.459999 \\
2.680956 \\
2.680956 \\
\\
9 \\
\\
\\
\\
0.000000 \\
4.554789\end{array}$ & $\begin{array}{c}5 \\
\\
\\
0.000000 \\
2.789168 \\
2.552569 \\
2.680956 \\
2.680956 \\
4.459999 \\
10 \\
\\
0.000000\end{array}$ & -20749.6483192 (+21.07 kcal/mole) \\
\hline $\begin{array}{c}\text { Charge } \\
4+\end{array}$ & $\begin{aligned} 1 \mathrm{G} \\
2 \mathrm{G} \\
3 \mathrm{G} \\
4 \mathrm{G} \\
5 \mathrm{G} \\
6 \mathrm{G} \\
7 \mathrm{G} \\
8 \mathrm{G} \\
9 \mathrm{G} \\
10 \mathrm{G} \\
\\
\\
6 \mathrm{G} \\
7 \mathrm{G} \\
8 \mathrm{G} \\
9 \mathrm{G} \\
10 \mathrm{G}\end{aligned}$ & $\begin{array}{c}1 \\
0.000000 \\
2.646288 \\
3.590314 \\
5.145747 \\
4.731938 \\
3.100448 \\
5.148227 \\
2.646747 \\
4.734263 \\
3.094664 \\
\\
6 \\
0.000000 \\
2.545338 \\
2.564124 \\
4.370787 \\
2.530883\end{array}$ & $\begin{array}{c}2 \\
0.000000 \\
2.646309 \\
3.641757 \\
3.861422 \\
3.868574 \\
4.670858 \\
3.302605 \\
2.565385 \\
2.565093 \\
\\
7 \\
\\
0.000000 \\
3.642233 \\
3.608757 \\
3.607695\end{array}$ & $\begin{array}{c}3 \\
\\
0.000000 \\
5.152655 \\
3.103679 \\
4.733335 \\
5.151108 \\
2.644082 \\
3.109966 \\
4.731348 \\
\\
8 \\
\\
\\
0.000000 \\
3.868316 \\
3.860775\end{array}$ & $\begin{array}{c}4 \\
\\
0.000000 \\
3.604589 \\
3.610294 \\
2.588050 \\
4.669682 \\
2.542458 \\
2.543788 \\
\\
9 \\
\\
\\
0.000000 \\
3.558231\end{array}$ & $\begin{array}{c}5 \\
\\
0.000000 \\
3.558231 \\
2.541608 \\
2.566349 \\
2.529383 \\
4.361350 \\
10 \\
\\
0.000000\end{array}$ & - 20748.4204394 (+28.94 kcal/mole) \\
\hline $\begin{array}{c}\text { Charge } \\
6+\end{array}$ & \multicolumn{6}{|c|}{ SPLIT } & {$[9+1]$} \\
\hline
\end{tabular}


C. $\mathrm{Ge}_{10}-2-1 \_C u b D i c a p-D_{4 h}$

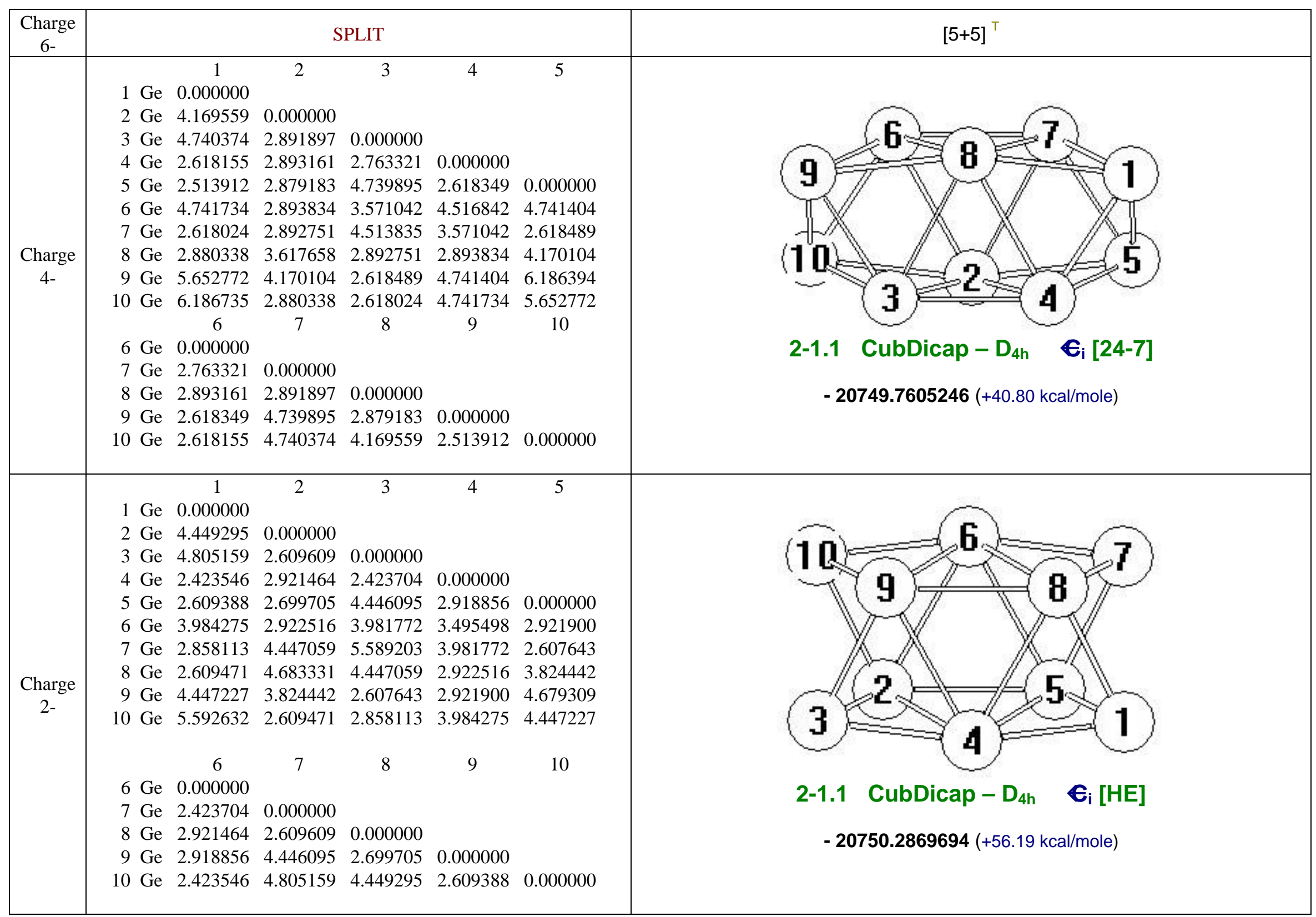




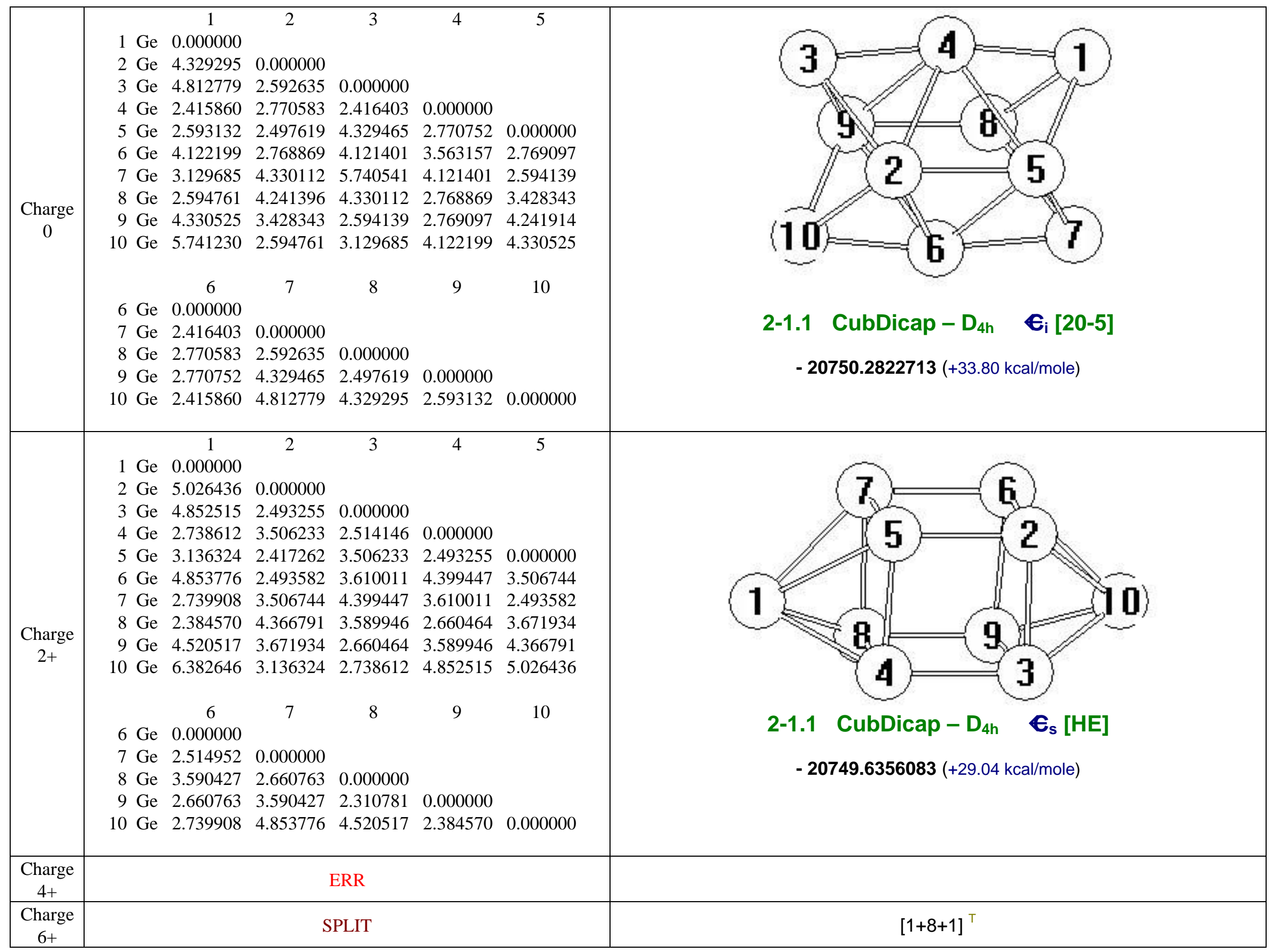


D. $\mathrm{Ge}_{10}-2-2 \_C u b D i c a p-\mathrm{C}_{2 v}$






\begin{tabular}{|c|c|c|c|c|c|c|c|}
\hline $\begin{array}{c}\text { Charge } \\
2-\end{array}$ & $\begin{aligned} 1 \mathrm{Ge} \\
2 \mathrm{Ge} \\
3 \mathrm{Ge} \\
4 \mathrm{Ge} \\
5 \mathrm{Ge} \\
6 \mathrm{Ge} \\
7 \mathrm{Ge} \\
8 \mathrm{Ge} \\
9 \mathrm{Ge} \\
10 \mathrm{Ge} \\
\\
\\
6 \mathrm{Ge} \\
7 \mathrm{Ge} \\
8 \mathrm{Ge} \\
9 \mathrm{Ge} \\
10 \mathrm{Ge}\end{aligned}$ & $\begin{array}{c}1 \\
0.000000 \\
4.208931 \\
3.989980 \\
2.589115 \\
2.821446 \\
4.187796 \\
2.540963 \\
2.819678 \\
4.209545 \\
2.542831 \\
\\
6 \\
0.000000 \\
2.588122 \\
4.187767 \\
2.589243 \\
2.589115\end{array}$ & $\begin{array}{c}2 \\
0.000000 \\
2.543318 \\
4.187767 \\
2.541451 \\
2.590341 \\
3.989722 \\
4.209622 \\
2.821272 \\
2.819678 \\
7 \\
\\
\\
0.000000 \\
2.541451 \\
2.822607 \\
2.821446\end{array}$ & $\begin{array}{c}3 \\
\\
0.000000 \\
2.589243 \\
2.822607 \\
4.189336 \\
4.209358 \\
2.821272 \\
2.541295 \\
4.209545 \\
\\
8 \\
\\
\\
0.000000 \\
2.543318 \\
4.208931\end{array}$ & $\begin{array}{c}4 \\
\\
0.000000 \\
2.588122 \\
5.333359 \\
4.188821 \\
2.590341 \\
4.189336 \\
4.187796 \\
\\
9 \\
\\
\\
\\
\\
0.000000 \\
3.989980\end{array}$ & $\begin{array}{c}5 \\
\\
\\
0.000000 \\
4.188821 \\
4.208792 \\
3.989722 \\
4.209358 \\
2.540963 \\
10\end{array}$ & 2-2.1 CubDicap $-\mathrm{C}_{2 \mathrm{v}} \quad \mathrm{G}_{2}[22-1]$ \\
\hline
\end{tabular}




\begin{tabular}{|c|c|c|c|c|c|c|c|}
\hline $\begin{array}{c}\text { Charge } \\
2+\end{array}$ & $\begin{aligned} 1 \mathrm{G} \\
2 \mathrm{G} \\
3 \mathrm{G} \\
4 \mathrm{G} \\
5 \mathrm{G} \\
6 \mathrm{G} \\
7 \mathrm{G} \\
8 \mathrm{G} \\
9 \mathrm{G} \\
10 \mathrm{G} \\
\\
\\
6 \mathrm{G} \\
7 \mathrm{G} \\
8 \mathrm{G} \\
9 \mathrm{G} \\
10 \mathrm{G}\end{aligned}$ & $\begin{array}{c}1 \\
0.000000 \\
4.732060 \\
4.759520 \\
2.667124 \\
2.611042 \\
4.732060 \\
2.611042 \\
2.667124 \\
4.759520 \\
4.337547 \\
\\
6 \\
0.000000 \\
2.530030 \\
3.522462 \\
2.483190 \\
2.667124\end{array}$ & $\begin{array}{c}2 \\
0.000000 \\
2.483190 \\
3.522462 \\
2.530030 \\
2.557643 \\
3.660888 \\
4.353077 \\
3.528666 \\
2.667124 \\
\\
7 \\
0.000000 \\
2.530030 \\
3.566667 \\
2.611042\end{array}$ & $\begin{array}{c}3 \\
\\
0.000000 \\
2.483190 \\
3.566667 \\
3.528666 \\
4.409974 \\
3.528666 \\
2.457439 \\
4.759520 \\
\\
8 \\
\\
\\
0.000000 \\
2.483190 \\
4.732060\end{array}$ & $\begin{array}{c}4 \\
\\
0.000000 \\
2.530030 \\
4.353077 \\
3.660888 \\
2.557643 \\
3.528666 \\
4.732060 \\
\\
9 \\
\\
\\
\\
0.000000 \\
4.759520\end{array}$ & $\begin{array}{c}5 \\
\\
\\
0.000000 \\
3.660888 \\
2.737304 \\
3.660888 \\
4.409974 \\
2.611042 \\
10\end{array}$ & $\begin{array}{l}\text { 2-2 CubDicap }-C_{2 v}[18-1] \\
-20749.6818918 \text { (+0.00 kcal/mole) }\end{array}$ \\
\hline $\begin{array}{c}\text { Charge } \\
4+\end{array}$ & $\begin{aligned} 1 \mathrm{G} \\
2 \mathrm{G} \\
3 \mathrm{G} \\
4 \mathrm{G} \\
5 \mathrm{G} \\
6 \mathrm{G} \\
7 \mathrm{G} \\
8 \mathrm{G} \\
9 \mathrm{G} \\
10 \mathrm{G} \\
\\
\\
\\
6 \mathrm{G} \\
7 \mathrm{G} \\
8 \mathrm{G} \\
9 \mathrm{G} \\
10 \mathrm{G}\end{aligned}$ & $\begin{array}{c}1 \\
0.000000 \\
5.030486 \\
5.023162 \\
2.696284 \\
2.782802 \\
5.030486 \\
2.782802 \\
2.696284 \\
5.023162 \\
4.733723 \\
\\
6 \\
0.000000 \\
2.643323 \\
3.620053 \\
2.465576 \\
2.967819\end{array}$ & $\begin{array}{c}2 \\
0.000000 \\
2.465576 \\
3.620053 \\
2.643323 \\
2.493506 \\
3.675485 \\
4.486455 \\
3.538792 \\
2.967819 \\
\\
7 \\
\\
0.000000 \\
2.467421 \\
3.656236 \\
2.768466\end{array}$ & $\begin{array}{c}3 \\
\\
0.000000 \\
2.706523 \\
3.656236 \\
3.538792 \\
4.486378 \\
3.821587 \\
2.584306 \\
5.096908 \\
\\
8 \\
\\
\\
0.000000 \\
2.706523 \\
4.982214\end{array}$ & $\begin{array}{c}4 \\
\\
0.000000 \\
2.467421 \\
4.486455 \\
3.668187 \\
2.816717 \\
3.821587 \\
4.982214 \\
\\
9\end{array}$ & $\begin{array}{c}5 \\
\\
0.000000 \\
3.675485 \\
2.615608 \\
3.668187 \\
4.486378 \\
2.768466 \\
10 \\
\\
0.000000\end{array}$ & 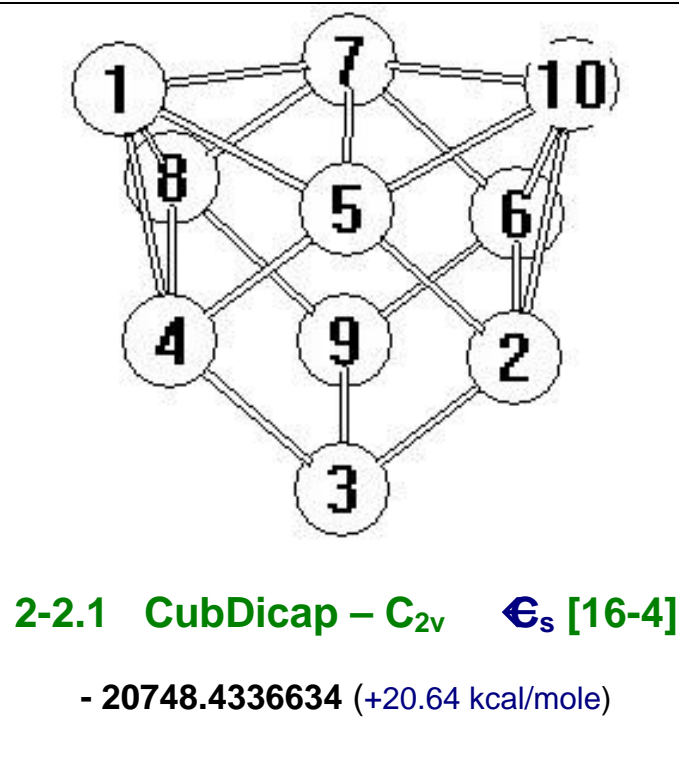 \\
\hline $\begin{array}{c}\text { Charge } \\
6+\end{array}$ & \multicolumn{6}{|c|}{ SPLIT } & {$[1+8+1]$} \\
\hline
\end{tabular}


E. $\mathrm{Ge}_{10}-3-1 \_P r P e n t a g-D_{5 h}$

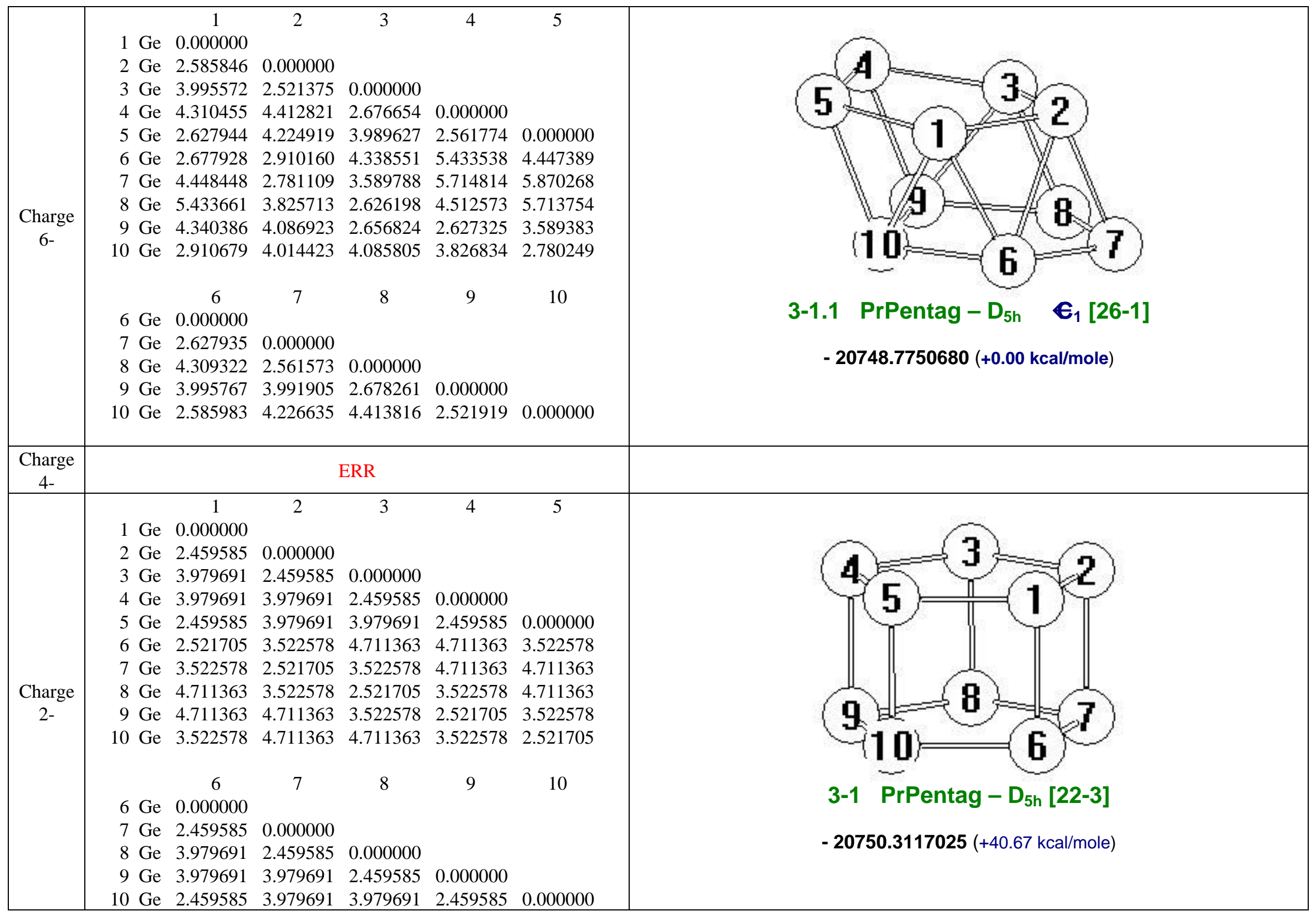




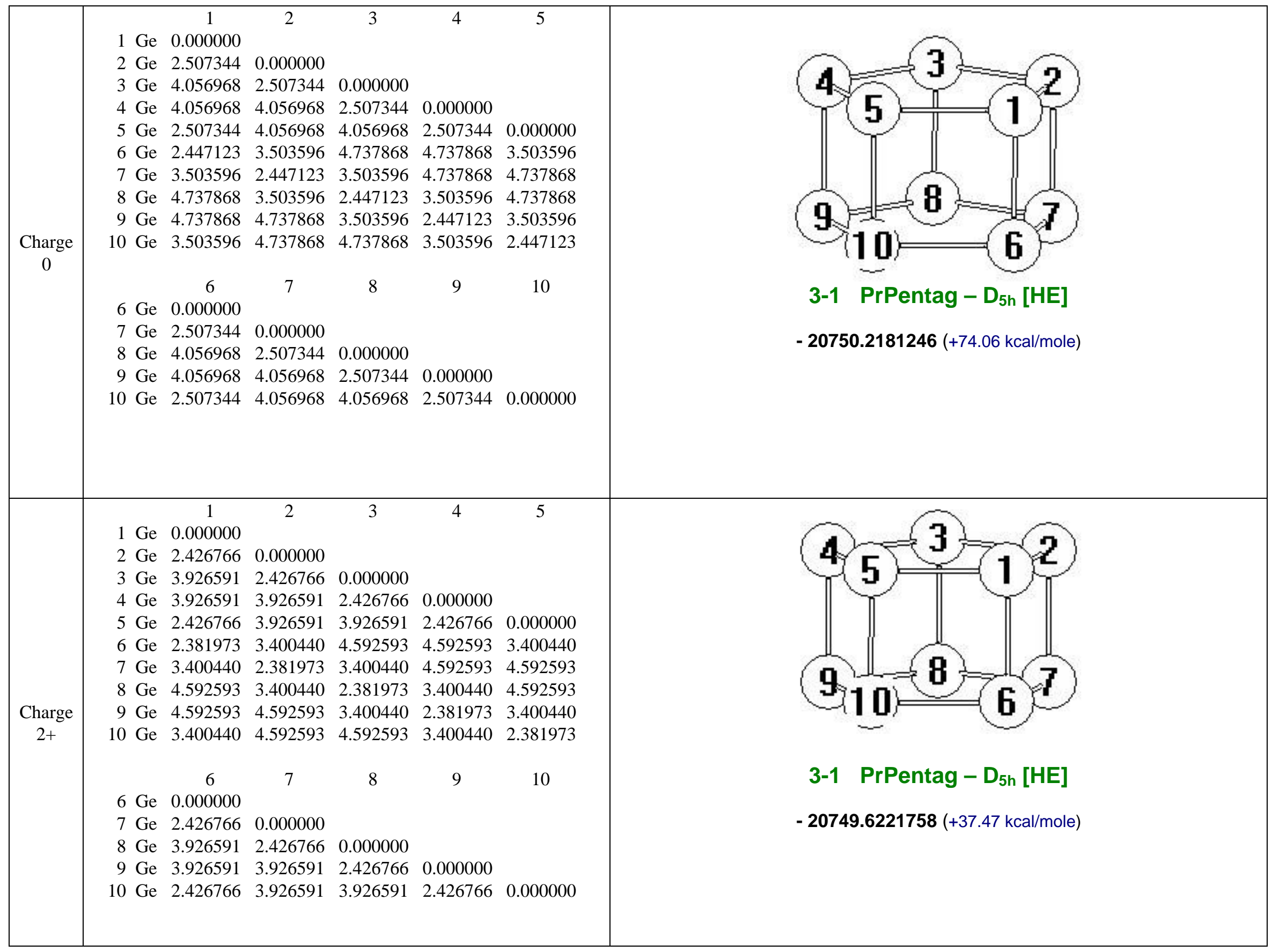




\begin{tabular}{|c|c|c|c|c|c|c|c|}
\hline $\begin{array}{c}\text { Charge } \\
4+\end{array}$ & $\begin{array}{r}1 \mathrm{Ge} \\
2 \mathrm{Ge} \\
3 \mathrm{Ge} \\
4 \mathrm{Ge} \\
5 \mathrm{Ge} \\
6 \mathrm{Ge} \\
7 \mathrm{Ge} \\
8 \mathrm{Ge} \\
9 \mathrm{Ge} \\
10 \mathrm{Ge} \\
\\
6 \mathrm{Ge} \\
7 \mathrm{Ge} \\
8 \mathrm{Ge} \\
9 \mathrm{Ge} \\
10 \mathrm{Ge}\end{array}$ & $\begin{array}{c}1 \\
0.000000 \\
2.541017 \\
3.706669 \\
4.866725 \\
2.546945 \\
2.480678 \\
3.551000 \\
4.451030 \\
5.540724 \\
3.543698 \\
\\
6 \\
0.000000 \\
2.541017 \\
3.706669 \\
4.866725 \\
2.546945\end{array}$ & $\begin{array}{c}2 \\
0.000000 \\
2.545725 \\
4.866726 \\
3.707697 \\
3.551000 \\
2.480302 \\
3.542891 \\
5.540629 \\
4.451625 \\
\\
7 \\
\\
0.000000 \\
2.545725 \\
4.866726 \\
3.707697\end{array}$ & $\begin{array}{c}3 \\
\\
0.000000 \\
2.591464 \\
2.856881 \\
4.451030 \\
3.542891 \\
2.447829 \\
3.692886 \\
3.761947 \\
\\
8 \\
\\
\\
0.000000 \\
2.591464 \\
2.856881\end{array}$ & $\begin{array}{c}4 \\
\\
0.000000 \\
2.590652 \\
5.540724 \\
5.540629 \\
3.692886 \\
2.827697 \\
3.692099 \\
\\
\quad \\
\\
\\
\\
\\
2.000000 \\
2.590652\end{array}$ & $\begin{array}{c}5 \\
\\
\\
0.000000 \\
3.543698 \\
4.451625 \\
3.761947 \\
3.692099 \\
2.447261 \\
10 \\
\\
\\
\\
0.000000\end{array}$ & 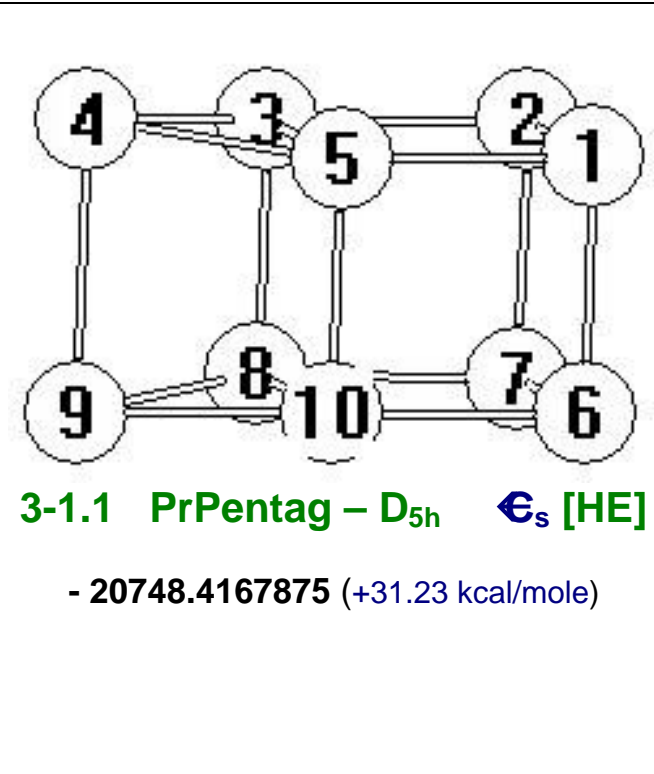 \\
\hline
\end{tabular}


F. $\mathrm{Ge}_{10}$ - 3-2_AntiPrPentag-D $\mathrm{D}_{5 \mathrm{~d}}$

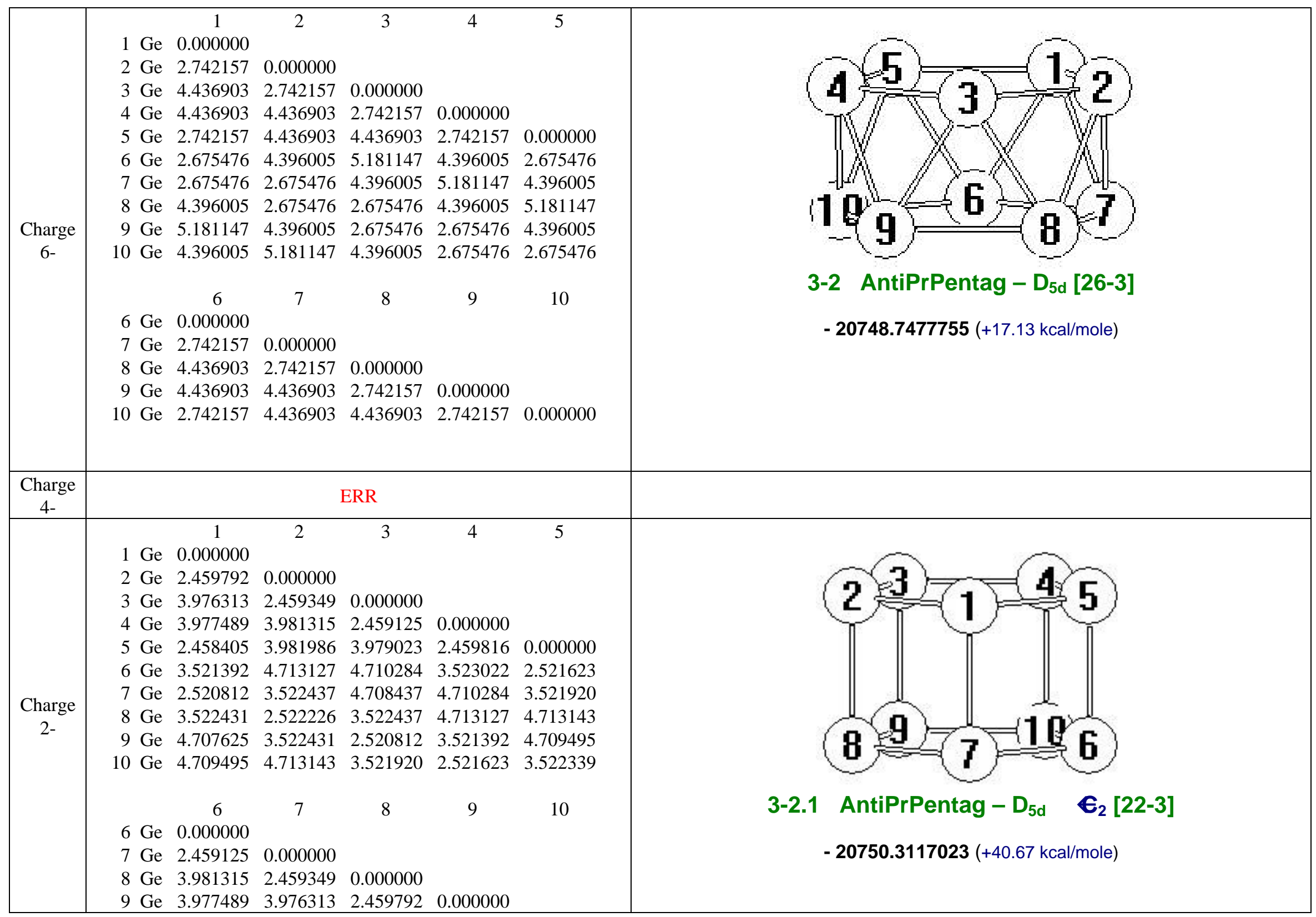




\begin{tabular}{|c|c|c|c|c|c|c|c|}
\hline & $10 \mathrm{Ge}$ & 2.459816 & 3.979023 & 3.981986 & 2.458405 & 0.000000 & \\
\hline $\begin{array}{c}\text { Charge } \\
2+\end{array}$ & & & & ERR & & & \\
\hline $\begin{array}{c}\text { Charge } \\
6+\end{array}$ & & & & ERR & & & \\
\hline
\end{tabular}


G. $\mathrm{Ge}_{10}-4-1 \_\mathrm{Ih}-2 \mathrm{vf}-\mathrm{D}_{2 \mathrm{~h}}$

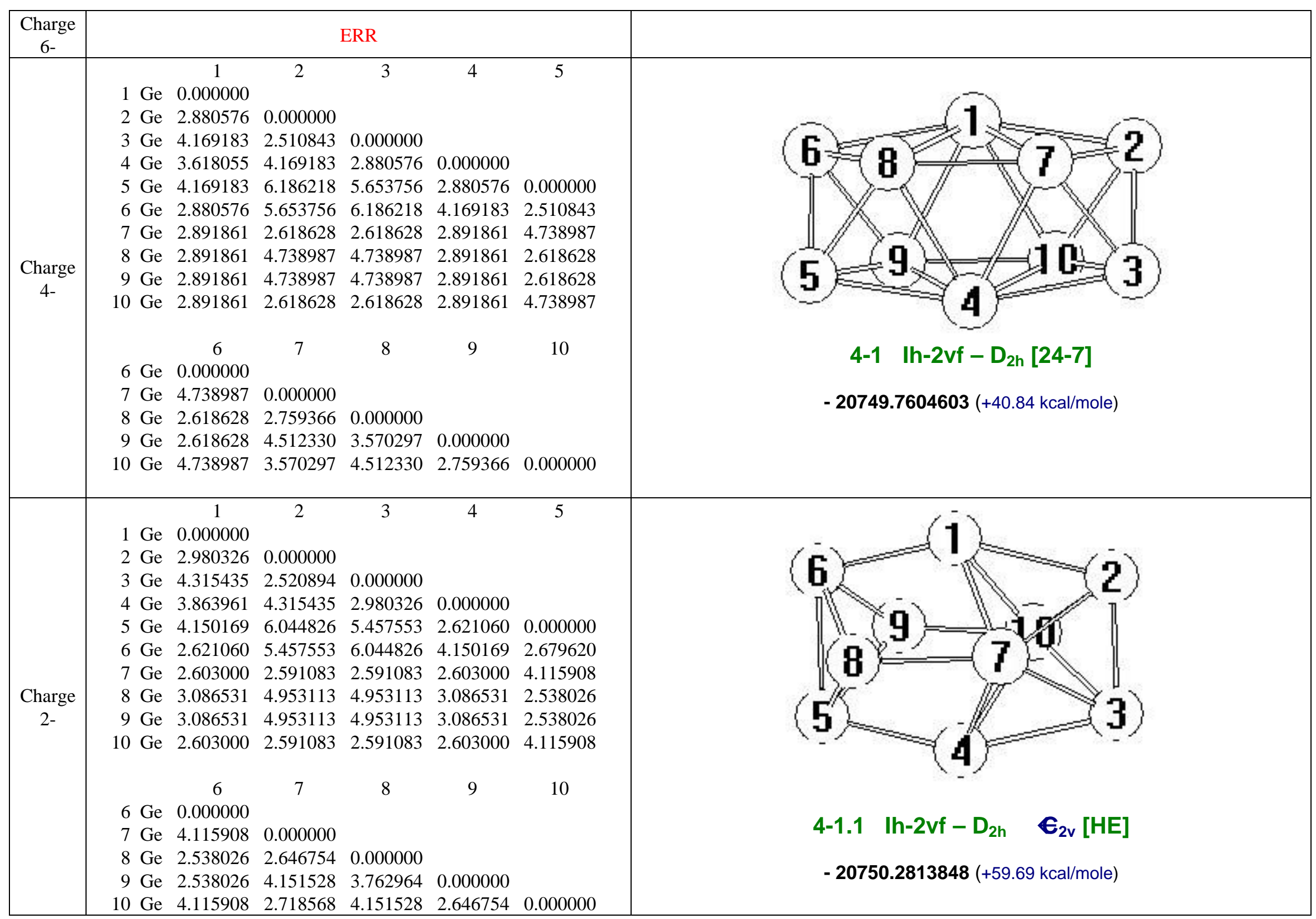




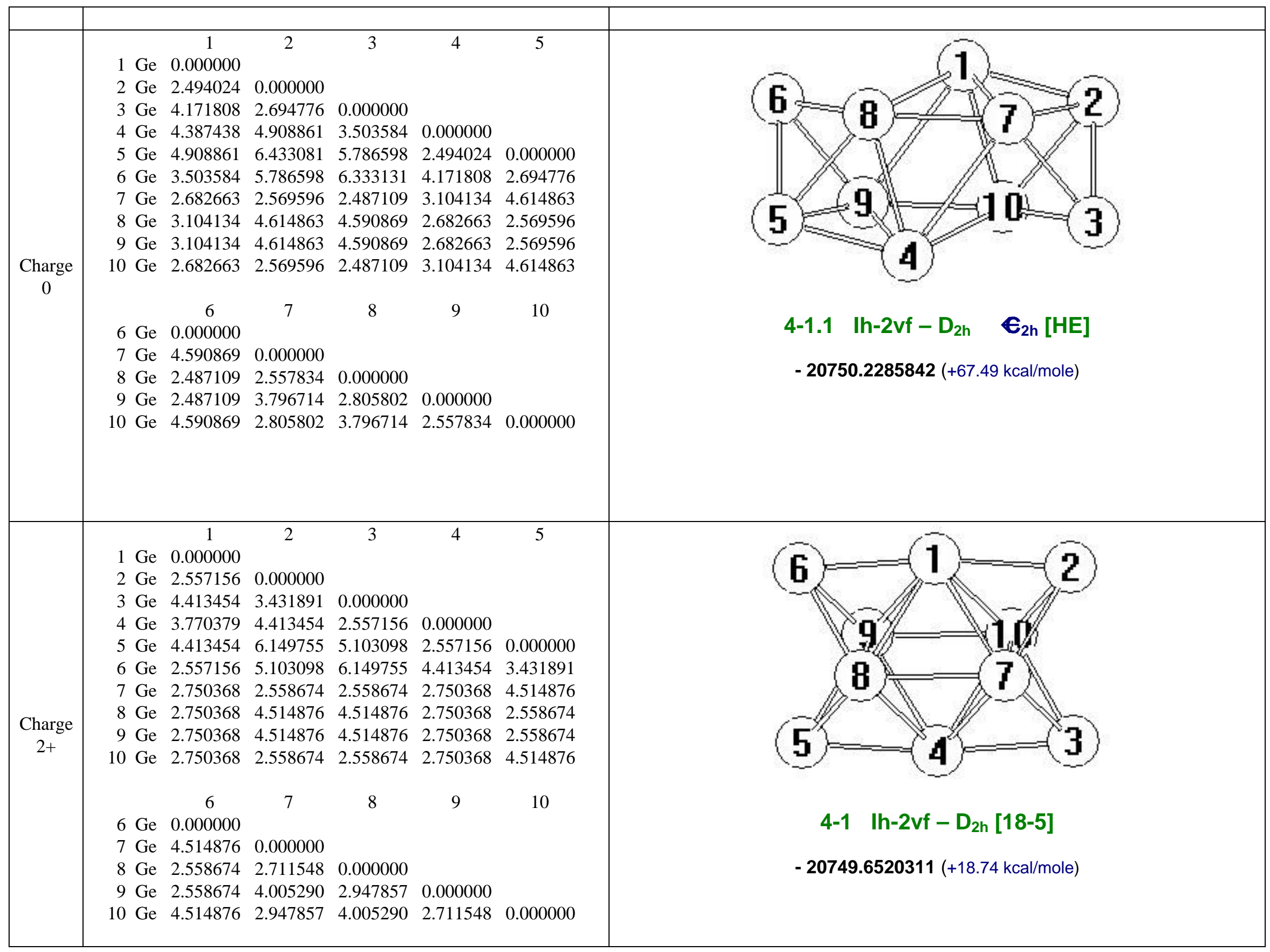




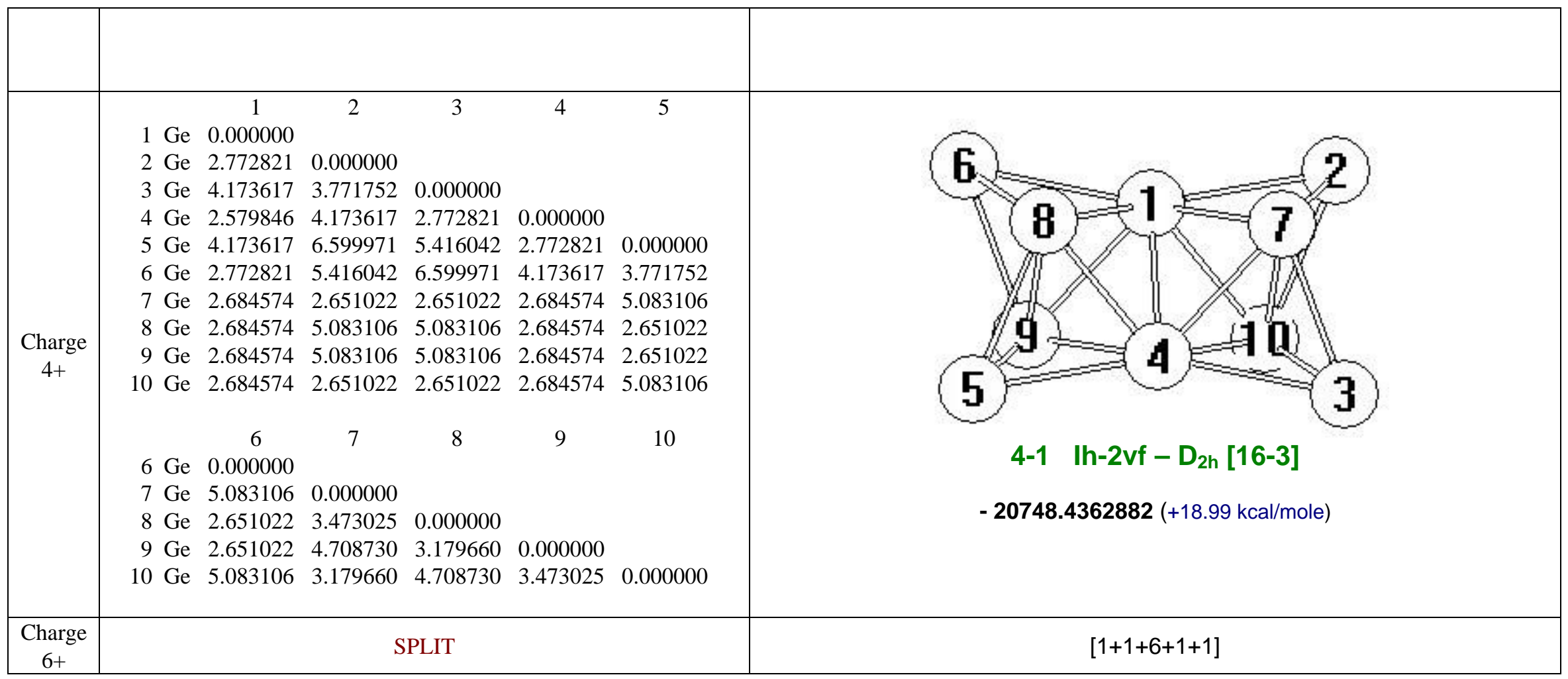


H. $\mathrm{Ge}_{10}-5-1 \_$AntiPrTetraDicap-D $\mathrm{D}_{4 \mathrm{~d}}$

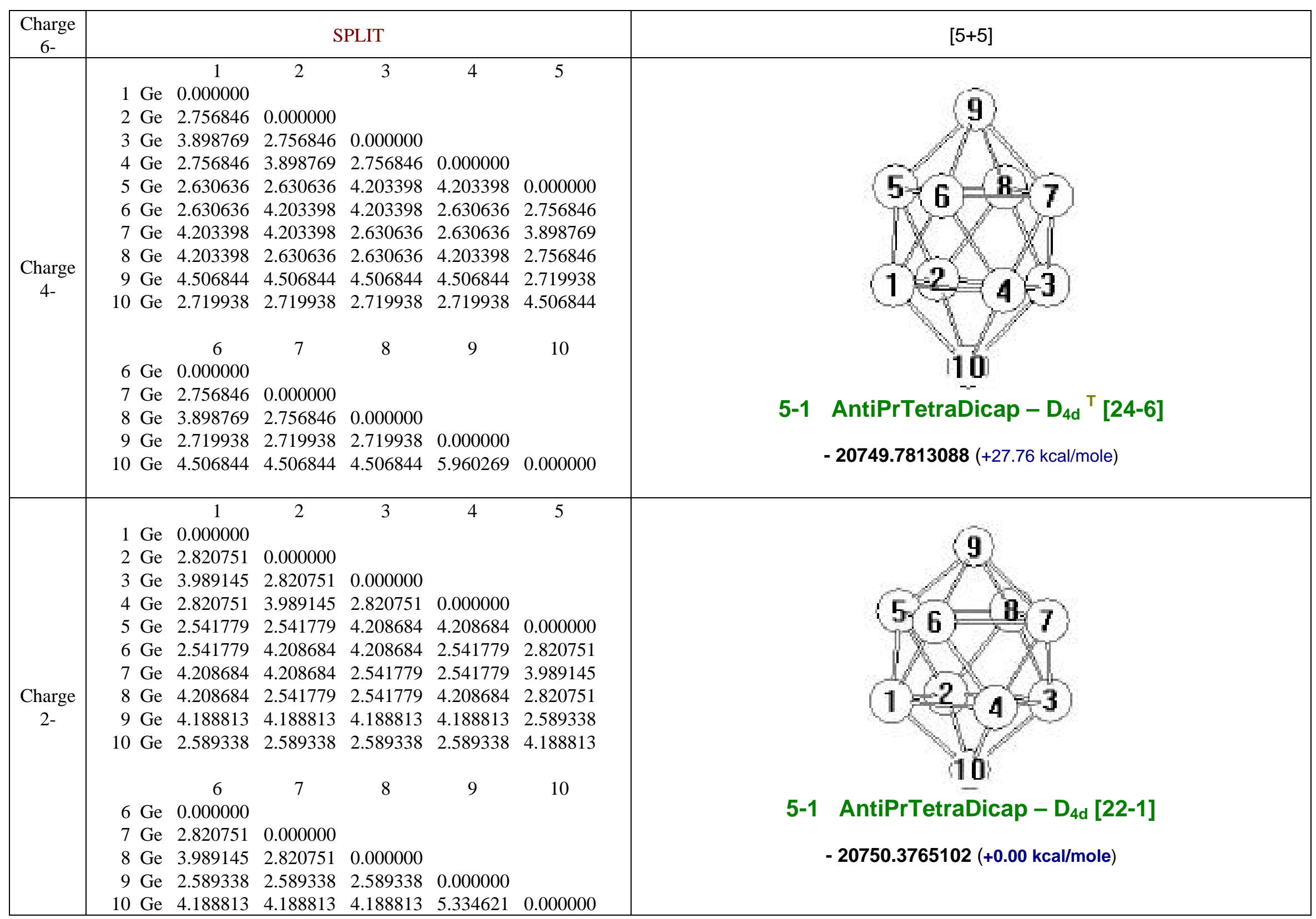




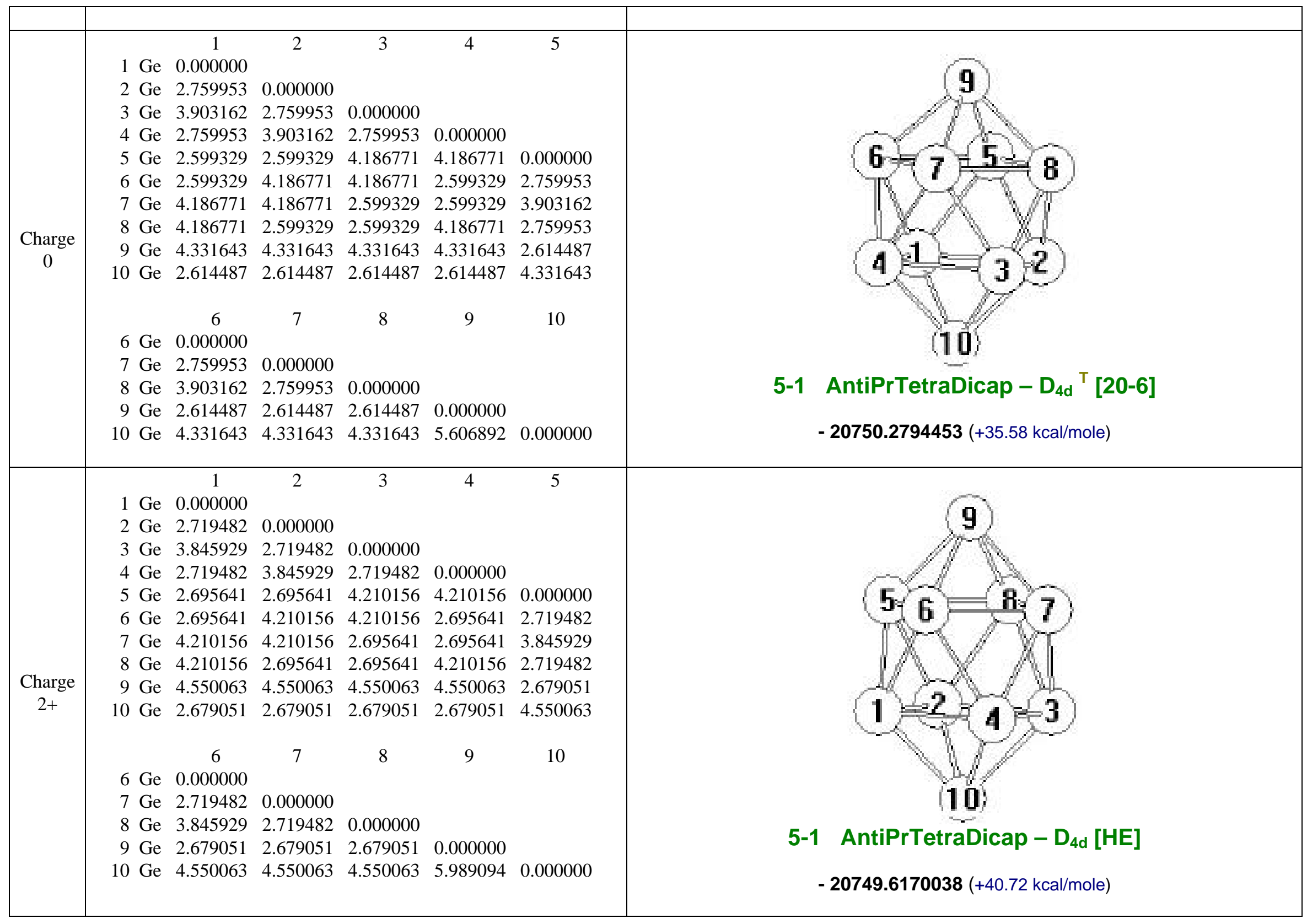




\begin{tabular}{|c|c|c|c|c|c|c|c|}
\hline $\begin{array}{c}\text { Charge } \\
4+\end{array}$ & $\begin{array}{r}1 \mathrm{Ge} \\
2 \mathrm{Ge} \\
3 \mathrm{Ge} \\
4 \mathrm{Ge} \\
5 \mathrm{Ge} \\
6 \mathrm{Ge} \\
7 \mathrm{Ge} \\
8 \mathrm{Ge} \\
9 \mathrm{Ge} \\
10 \mathrm{Ge} \\
\\
\\
6 \mathrm{Ge} \\
7 \mathrm{Ge} \\
8 \mathrm{Ge} \\
9 \mathrm{Ge} \\
10 \mathrm{Ge}\end{array}$ & $\begin{array}{c}1 \\
0.000000 \\
2.902896 \\
4.940246 \\
2.903773 \\
2.597843 \\
4.584957 \\
4.540089 \\
4.585846 \\
5.230709 \\
2.965600 \\
6 \\
0.000000 \\
2.903972 \\
4.939974 \\
2.965022 \\
5.231387\end{array}$ & $\begin{array}{c}2 \\
0.000000 \\
2.903773 \\
2.804736 \\
2.656648 \\
4.539756 \\
2.657423 \\
2.598858 \\
4.247882 \\
2.644574 \\
\\
7 \\
\\
0.000000 \\
2.903547 \\
2.644970 \\
4.248143\end{array}$ & $\begin{array}{c}3 \\
\\
0.000000 \\
2.902896 \\
4.540089 \\
4.585846 \\
2.597843 \\
4.584957 \\
5.230709 \\
2.965600 \\
8\end{array}$ & $\begin{array}{c}4 \\
\\
0.000000 \\
2.657423 \\
2.598858 \\
2.656648 \\
4.539756 \\
4.247882 \\
2.644574 \\
9\end{array}$ & $\begin{array}{c}0.000000 \\
2.903547 \\
2.806262 \\
2.903972 \\
2.644970 \\
4.248143 \\
10\end{array}$ & $\begin{array}{l}\text { 5-1 AntiPrTetraDicap }-D_{4 d} \quad \in_{2}[16-1] \\
-20748.4665524(+0.00 \mathrm{kcal} / \mathrm{mole})\end{array}$ \\
\hline
\end{tabular}


I. $\mathrm{Ge}_{10}-6-1 \_$Bisdisf- $\mathrm{D}_{2 \mathrm{~d}}$

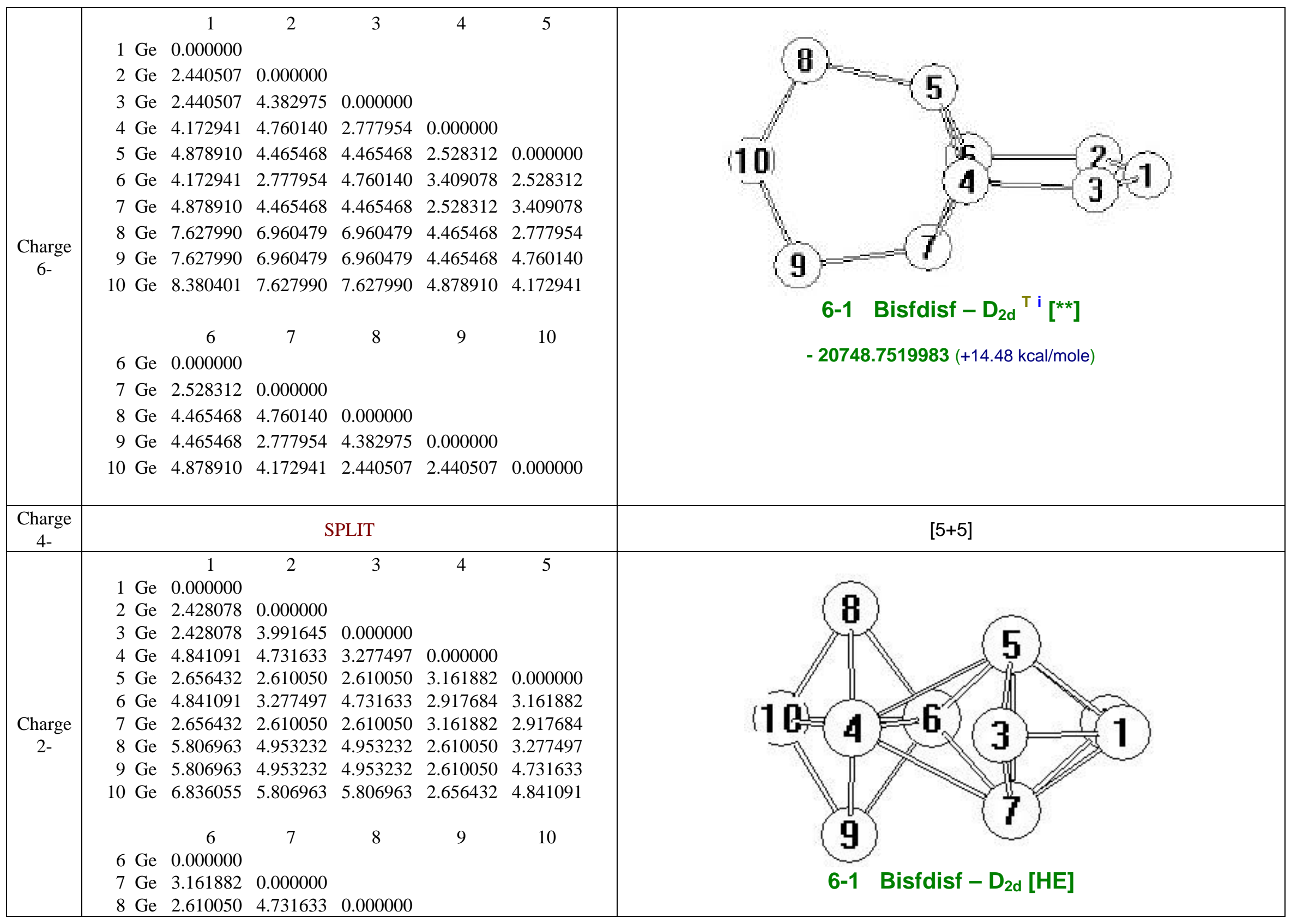




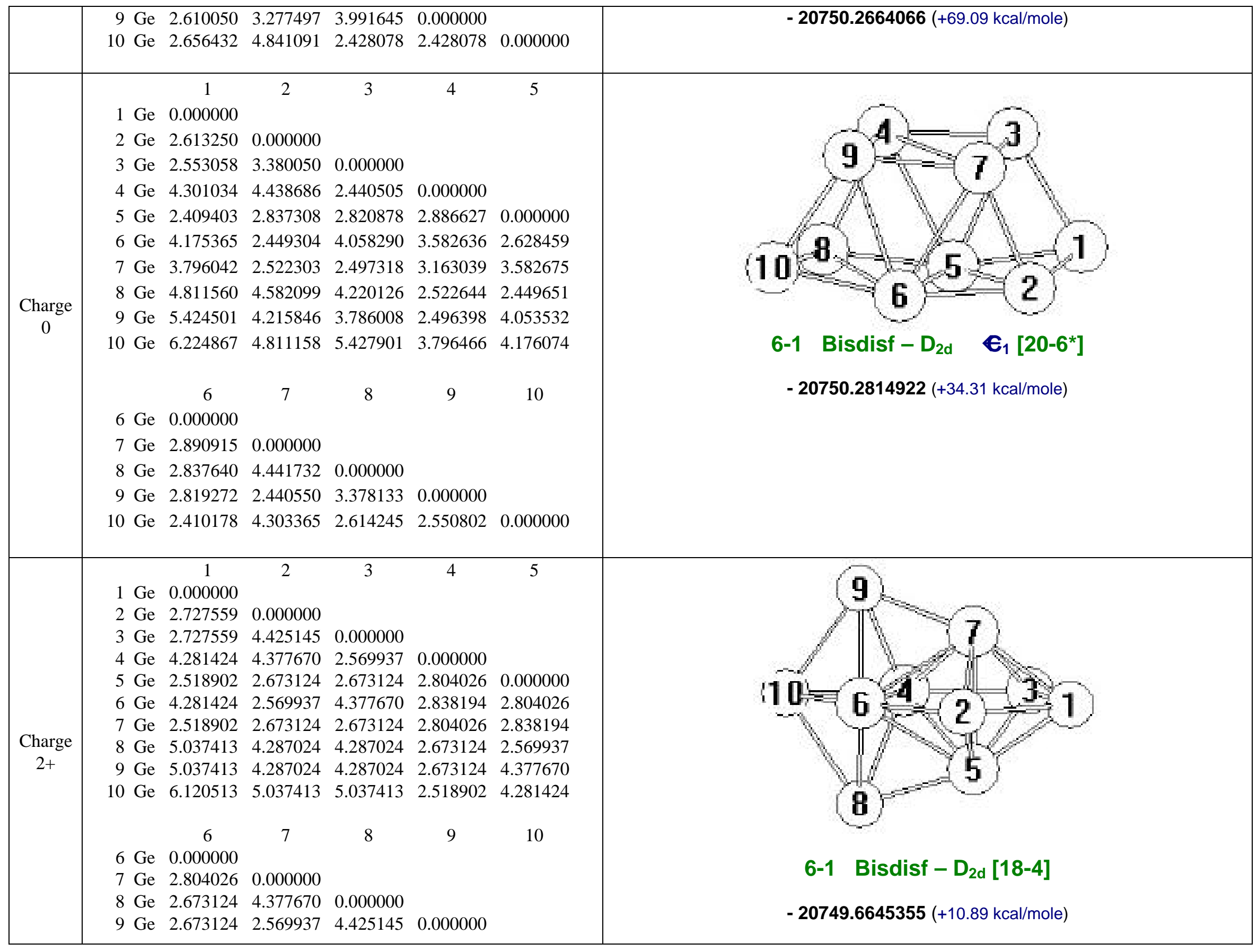




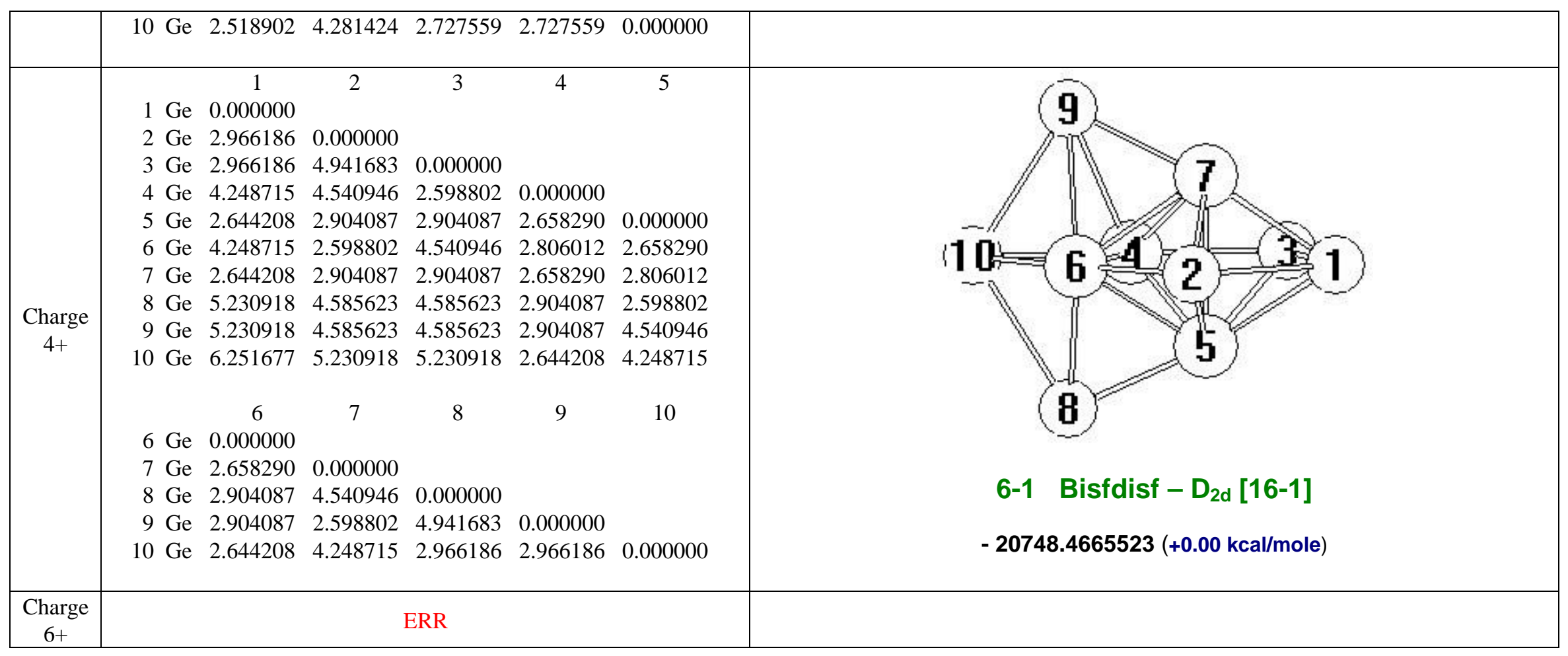


J. $\mathrm{Ge}_{10}-7-1 \_C e n t a u r-\mathrm{C}_{3 \mathrm{v}}$

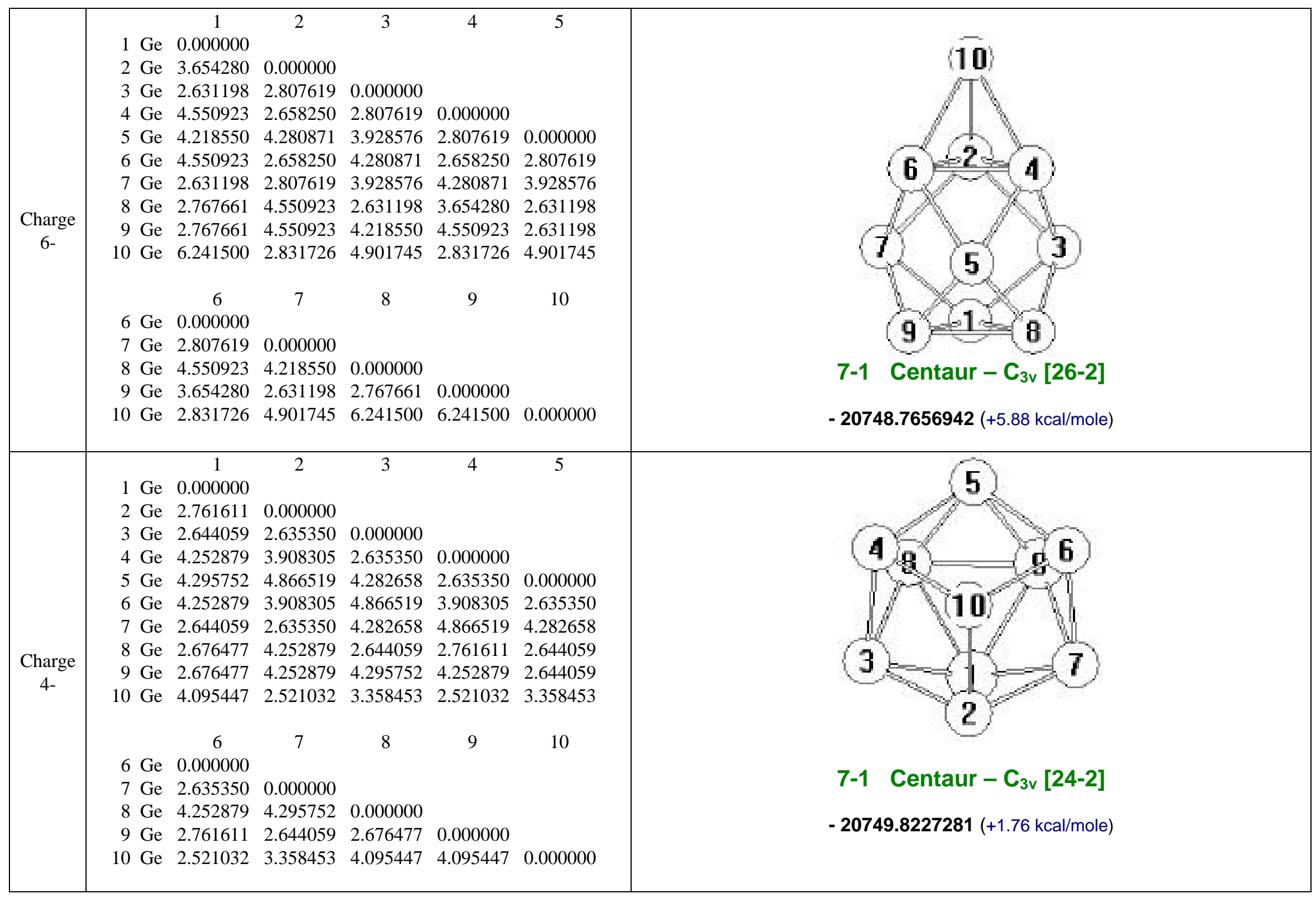




\begin{tabular}{|c|c|c|c|c|c|c|c|}
\hline $\begin{array}{c}\text { Charge } \\
2-\end{array}$ & $\begin{aligned} & 1 \mathrm{Ge} \\
& 2 \mathrm{Ge} \\
& 3 \mathrm{Ge} \\
& 4 \mathrm{Ge} \\
& 5 \mathrm{Ge} \\
& 6 \mathrm{Ge} \\
& 7 \mathrm{Ge} \\
& 8 \mathrm{Ge} \\
& 9 \mathrm{Ge} \\
& 10 \mathrm{Ge} \\
& \\
& 6 \mathrm{Ge} \\
& 7 \mathrm{Ge} \\
& 8 \mathrm{Ge} \\
& 9 \mathrm{Ge} \\
& 10 \mathrm{Ge}\end{aligned}$ & $\begin{array}{c}1 \\
0.000000 \\
2.784172 \\
2.616478 \\
4.175670 \\
4.213183 \\
4.175670 \\
2.616478 \\
2.630999 \\
2.630999 \\
4.242648 \\
\\
6 \\
0.000000 \\
2.546932 \\
4.175670 \\
2.784172 \\
2.462536\end{array}$ & $\begin{array}{c}2 \\
0.000000 \\
2.546932 \\
3.680962 \\
4.663014 \\
3.680962 \\
2.546932 \\
4.175670 \\
4.175670 \\
2.462536 \\
\\
7 \\
\\
0.000000 \\
4.213183 \\
2.616478 \\
3.387095\end{array}$ & $\begin{array}{c}3 \\
\\
0.000000 \\
2.546932 \\
4.144797 \\
4.663014 \\
4.144797 \\
2.616478 \\
4.213183 \\
3.387095 \\
\\
\end{array}$ & $\begin{array}{c}4 \\
\\
0.000000 \\
2.546932 \\
3.680962 \\
4.663014 \\
2.784172 \\
4.175670 \\
2.462536 \\
\\
9 \\
\\
\\
\\
0.000000 \\
4.242648\end{array}$ & $\begin{array}{c}5 \\
\\
\\
0.000000 \\
2.546932 \\
4.144797 \\
2.616478 \\
2.616478 \\
3.387095 \\
10\end{array}$ & $\begin{array}{l}\text { 7-1 Centaur }-\mathrm{C}_{3 \mathrm{v}}{ }^{\top}[22-2] \\
-\mathbf{2 0 7 5 0 . 3 4 9 8 4 3 3}(+16.73 \mathrm{kcal} / \mathrm{mole})\end{array}$ \\
\hline $\begin{array}{c}\text { Charge } \\
0\end{array}$ & $\begin{aligned} & 1 \\
& 2 \mathrm{Ge} \\
& 3 \mathrm{Ge} \\
& 3 \mathrm{Ge} \\
& 4 \mathrm{Ge} \\
& 5 \mathrm{Ge} \\
& 6 \mathrm{Ge} \\
& 7 \mathrm{Ge} \\
& 8 \mathrm{Ge} \\
& 9 \mathrm{Ge} \\
& 10 \mathrm{Ge} \\
& \\
& \\
& 6 \mathrm{Ge} \\
& 7 \mathrm{Ge} \\
& 8 \mathrm{Ge} \\
& 9 \mathrm{Ge} \\
& 10 \mathrm{Ge}\end{aligned}$ & $\begin{array}{c}1 \\
0.000000 \\
3.123414 \\
2.596958 \\
4.540198 \\
3.979573 \\
4.540198 \\
2.596958 \\
2.644613 \\
2.644613 \\
4.223013 \\
\\
6 \\
0.000000 \\
2.478604 \\
4.540198 \\
3.123414 \\
2.546258\end{array}$ & $\begin{array}{c}2 \\
0.000000 \\
2.478604 \\
4.105584 \\
4.501050 \\
4.105584 \\
2.478604 \\
4.540198 \\
4.540198 \\
2.546258 \\
\\
7 \\
\\
0.000000 \\
3.979573 \\
2.596958 \\
2.864827\end{array}$ & $\begin{array}{c}3 \\
\\
0.000000 \\
2.478604 \\
3.438238 \\
4.501050 \\
3.438238 \\
2.596958 \\
3.979573 \\
2.864827 \\
8\end{array}$ & $\begin{array}{c}4 \\
\\
0.000000 \\
2.478604 \\
4.105584 \\
4.501050 \\
3.123414 \\
4.540198 \\
2.546258 \\
\\
9 \\
\\
\\
0.000000 \\
4.223013\end{array}$ & $\begin{array}{c}5 \\
\\
0.000000 \\
2.478604 \\
3.438238 \\
2.596958 \\
2.596958 \\
2.864827 \\
10\end{array}$ & - 20750.3308891 (+3.30 kcal/mole) \\
\hline
\end{tabular}




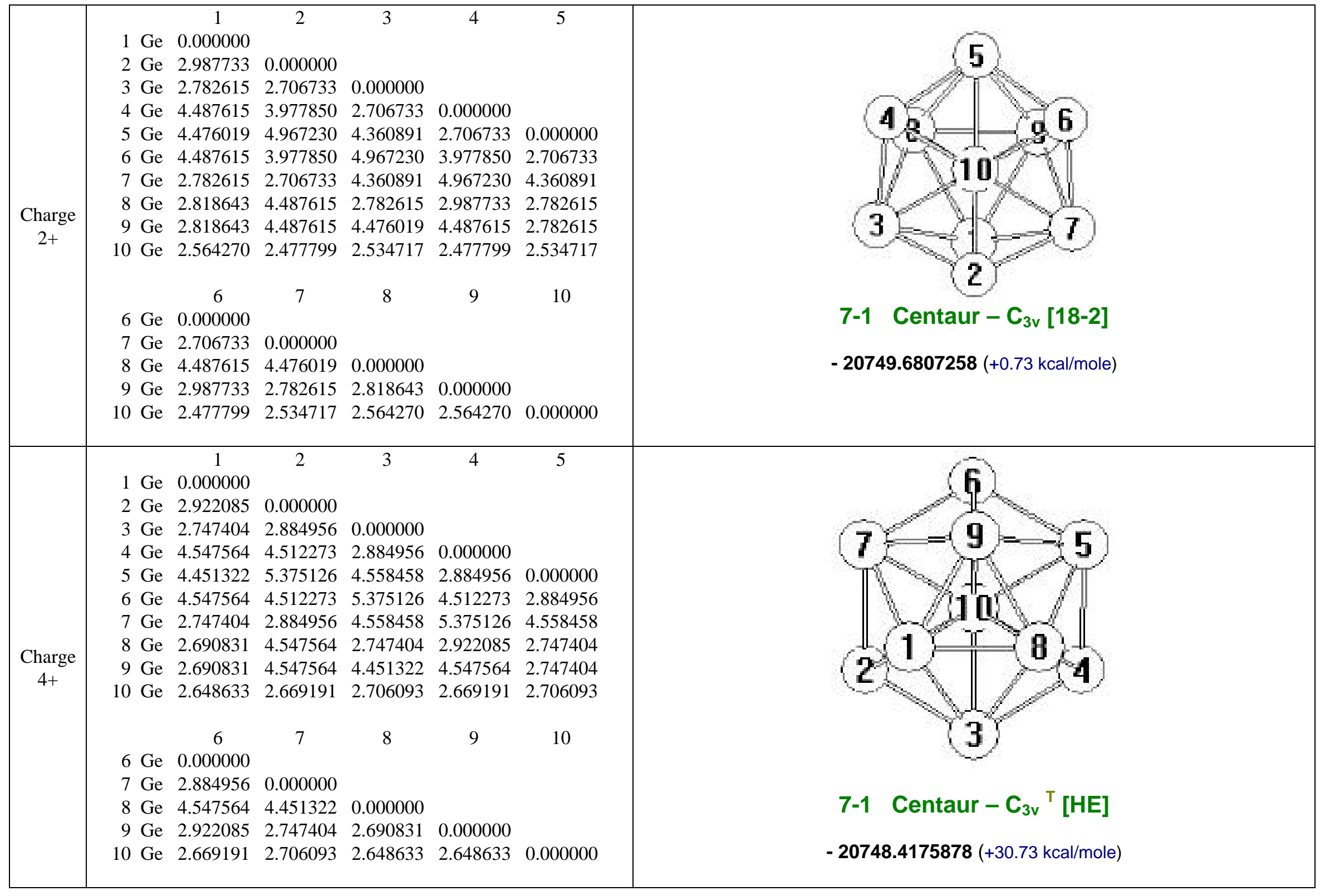




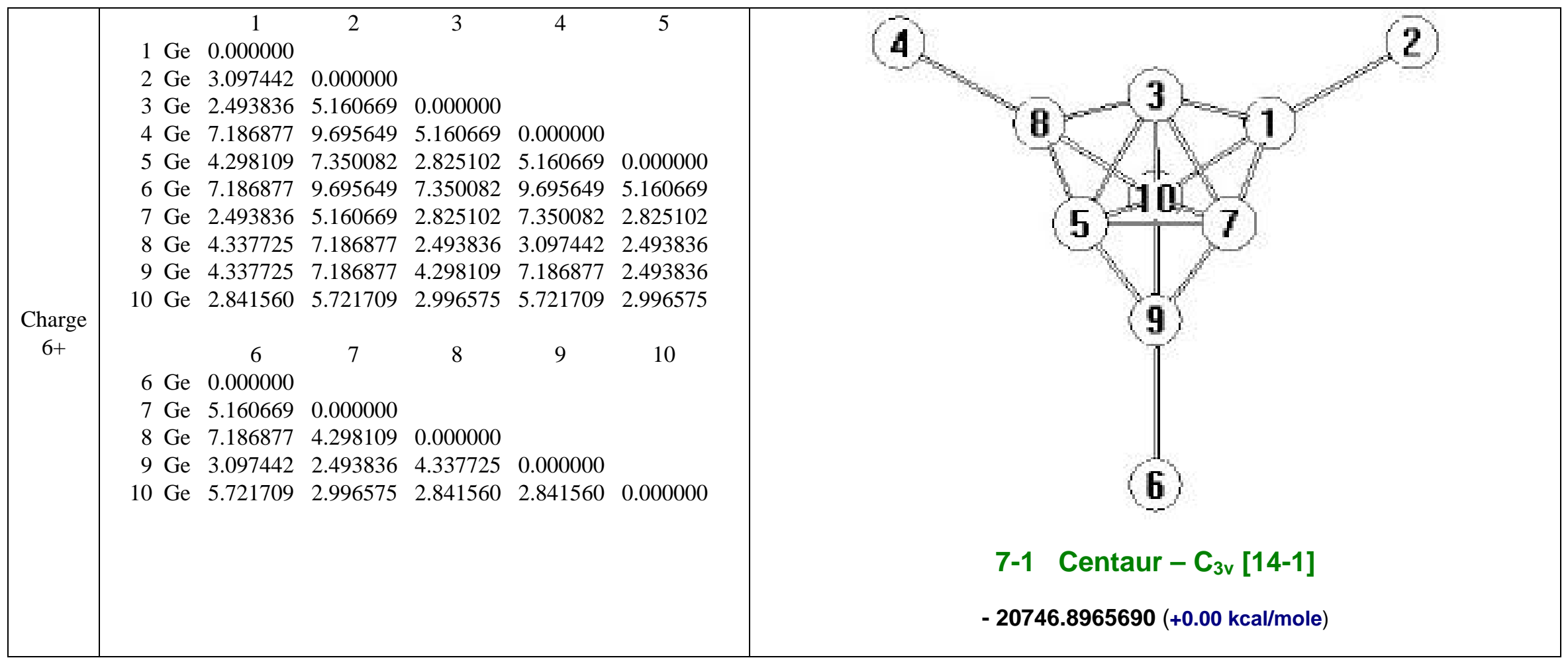

